\title{
Distributed optical fibre audible frequency sensor
}

\author{
Ali Masoudi ${ }^{1}$, Mohammad Belal ${ }^{1}$ and Trevor P. Newson ${ }^{1}$ \\ ${ }^{1}$ ORC, University of Southampton, Southampton, SO17 1BJ, UK
}

\begin{abstract}
A distributed optical fibre sensor is demonstrated which is capable of quantifying acoustic and dynamic strain disturbances along a $1 \mathrm{~km}$ sensing fibre. A phase-OTDR technique is used to detect the dynamic perturbations using the phase-difference between the backscattered light from two separate sections of the sensing fibre. The demonstrated sensor detects multiple dynamic perturbations simultaneously within a frequency range of $200 \mathrm{~Hz}$ to $5000 \mathrm{~Hz}$ with a frequency resolution of $10 \mathrm{~Hz}$ and a spatial resolution of $1 \mathrm{~m}$.
\end{abstract}

Keyword: Acoustic sensing, OTDR, Dynamic strain sensing

\section{INTRODUCTION}

Following the early demonstration in 1977 by Cole et al. ${ }^{[1]}$ and Bruno et al. ${ }^{[2]}$ that sound could be detected using optical fibres, much of the subsequent research activity has focused on developing optical fibre hydrophones. The majority of such devices have utilized multiple fibre optic Mach-Zehnder interferometers (MZI) for detecting the acoustically induced phase perturbations. Such systems are described as quasi-distributed sensors, as measurements of the acoustic information is collected at discrete points. An early reported distributed sensor proposed by Kurmer et al. ${ }^{\text {[3] }}$ used a Sagnac interferometer to detect acoustic vibration anywhere along a $5 \mathrm{~km}$ of fibre. Whilst this technique is capable of detecting the location of a single acoustic perturbation along the sensing fibre, it does not allow multiple acoustic disturbances to be monitored.

The first distributed acoustic optical fibre sensor capable of detecting multiple disturbances was introduced in $1991^{[4]}$. This sensor exploited the changes in the backscattered coherent Rayleigh noise (CRN) when the fibre is acoustically perturbed. Although the technique is capable of providing the location and the frequency of the acoustic sources along the sensing fibre, it is unable to measure the magnitude of the disturbances. An optical time domain reflectometry (OTDR) system which overcame this problem and was capable of quantifying dynamic disturbances was proposed by Posey et al. ${ }^{[5]}$ but the technique was limited to monitoring dynamic strain at only one point at any one time.

The first distributed optical fibre dynamic strain sensor capable of quantifying dynamic strains simultaneously along the entire sensing fibre was reported by Masoudi et al. ${ }^{[6]}$. The minimum detectable frequency of $200 \mathrm{~Hz}$ was limited by low frequency noise in the system and the signal processing procedure. Furthermore, the sensor could not be used to accurately monitor the amplitude of non-planar acoustic waves due to its $2 \mathrm{~m}$ spatial resolution.

In this paper, by optimizing the concept used for dynamic strain sensor, a DOFS is demonstrated for the first time which is capable of simultaneously detecting multiple audio frequencies $(200 \mathrm{~Hz}$ to $5 \mathrm{kHz})$ along the sensing fibre. In addition to improved frequency and spatial resolution, the signal processing procedure was modified to improve the signal to noise ratio (SNR).

The principle of operation of the proposed distributed acoustic sensor is based on the variation of the phase of the backscattered Rayleigh from two sections of the sensing fibre. This concept is illustrated in figure 1 . If the phase of the backscattered light from the scattering centre $A$ is $\varphi_{1}$ and that of the scattering centre $B$, sitting $L$ meters apart from the scattering centre $A$, is $\varphi_{2}$, the phase difference between the two centres remains constant if there is no disturbance between the two centres. However, any perturbation which changes the separation of the two centres results in a variation of phase difference between $\mathrm{A}$ and $\mathrm{B}$. This variation of phase difference, $\Delta \phi$, is converted to an intensity change by an unbalanced Mach-Zehnder interferometer (MZI). In addition, to eliminate signal fading problem of the MZI transfer function, a $3 \times 3$ coupler is used at the output end of the MZI ${ }^{[7]}$. The output intensity of the three arms of this configuration is ${ }^{[6]}$

$$
\begin{aligned}
& I_{1}=I_{0}[A+B \cos (\Delta \phi)] \\
& I_{2}=I_{0}\left[A+B \cos \left(\Delta \phi+\frac{2 \pi}{3}\right)\right] \\
& I_{3}=I_{0}\left[A+B \cos \left(\Delta \phi-\frac{2 \pi}{3}\right)\right]
\end{aligned}
$$


where $A$ and $B$ are constant, $I_{0}$ is the intensity of the input signal, and $\Delta \phi=\varphi_{1}-\left(\varphi_{2}+\Delta \varphi\right)$. Using differentiate and cross-multiplying demodulation technique, the outputs of the MZI in equation (1) can be combined to extract the phase information given by ${ }^{[8]}$

where $\Phi$ is the output of the phase detector.

$$
\Phi=\sqrt{3} \Delta \phi
$$

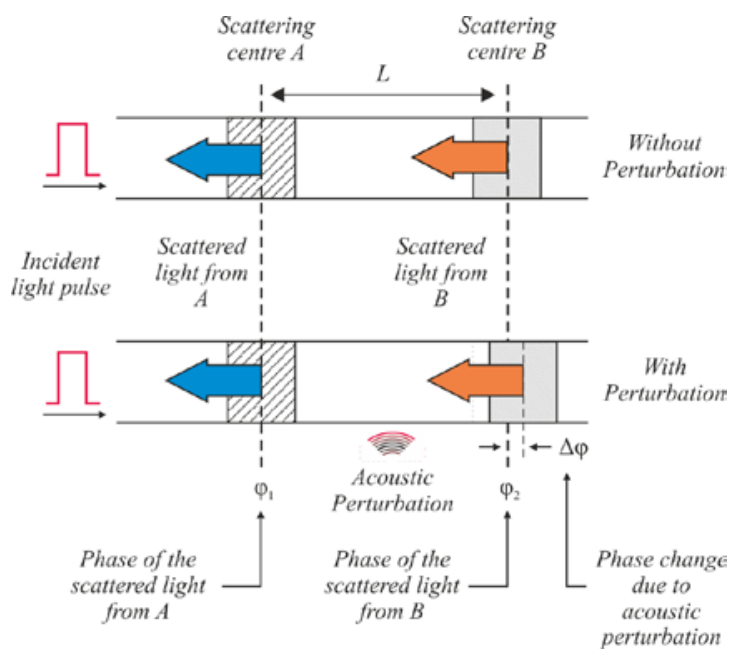

Figure 1: Principle of the dynamic strain sensor using $\varphi$-OTDR. This figure represents the behavior of two groups of scatteres inside the sensing fibre before and after external acoustic perturbation.

\section{EXPERIMENT}

The experimental setup is shown in figure 2. A $1550 \mathrm{~nm}$ distributed feedback (DFB) laser diode was directly modulated to generate $10 \mathrm{~ns}$ pulse with a peak power of $10 \mathrm{~mW}$ and a repetition rate of $20 \mu \mathrm{s}$. The pulse was amplified to a peak power of $6 \mathrm{~W}$ by a $28 d B$ gain Erbium-doped fibre amplifier (EDFA1). The amplified spontaneous emission (ASE) from EDFA1 was filtered by a fibre Bragg grating (FBG1) (Reflectivity $99 \% ; \Delta \lambda=3 n m ; \lambda=$ $1550.7 \mathrm{~nm}$ ) and the amplified pulse was launched into the sensing fibre via circulator C2.

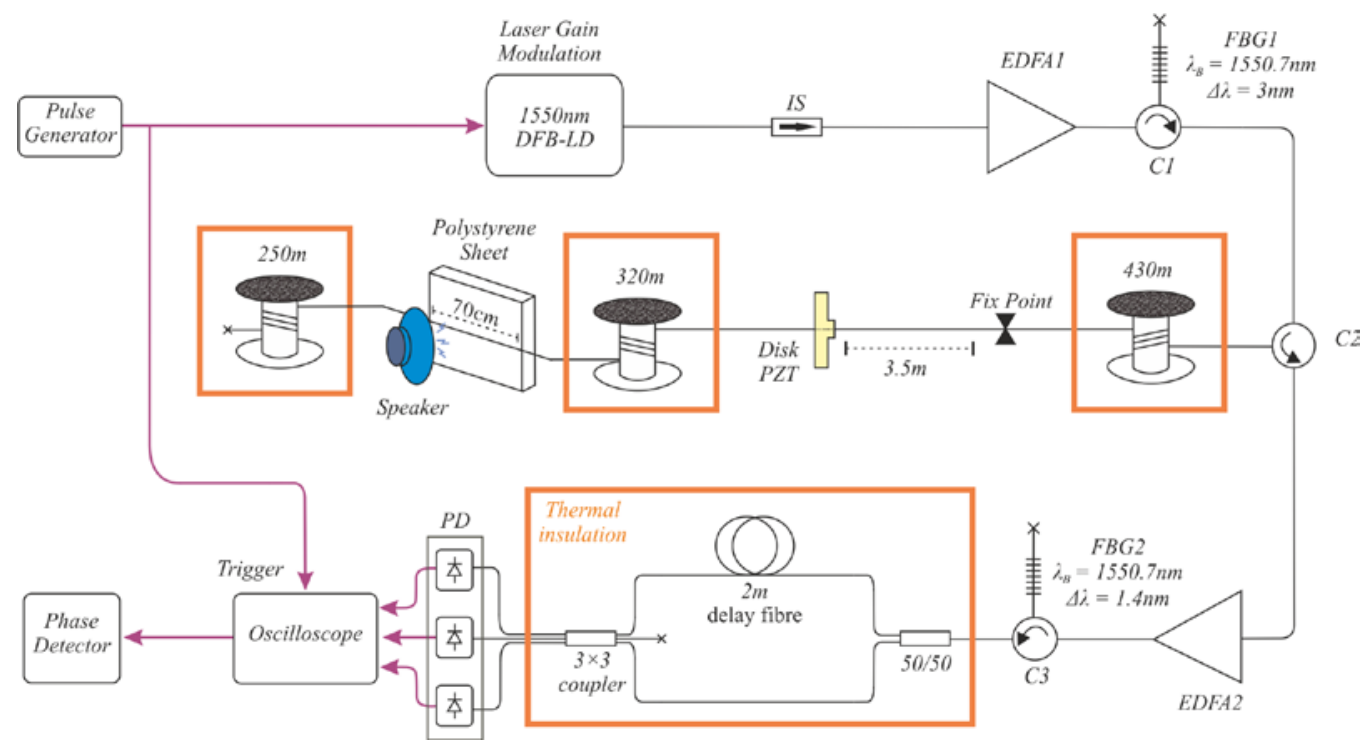

Figure 2: Experimental Setup. DFB-LD: distributed feedback laser source, IS: isolator, EDFA: erbium-doped fibre amplifier, PD: photodetector, FBG: fibre Bragg grating, C: circulator. 
The $1 \mathrm{~km}$ sensing fibre comprised of five sections of standard single mode fibre (SSMF) including a $3.5 \mathrm{~m}$ section where dynamic strain was imposed using a disk PZT and a $70 \mathrm{~cm}$ section where the acoustic perturbation was applied via a loud-speaker. To improve the acoustic sensitivity of the $70 \mathrm{~cm}$ section, the fibre was attached to a $700 \mathrm{~mm} \times 450 \mathrm{~mm} \times 25 \mathrm{~mm}$ polystyrene sheet. A $320 \mathrm{~m}$ of thermally and acoustically insulated fibre was used to separate the two previously mentioned sections while a further $430 \mathrm{~m}$ and $250 \mathrm{~m}$ of thermally and acoustically insulated fibres were used before and after the $3.5 \mathrm{~m}$ and the $70 \mathrm{~cm}$ sections respectively.

The Rayleigh backscattered signal was amplified using a $25 d B$ gain optical amplifier (EDFA2). The ASE from the optical amplifier was filter by a second fibre Bragg grating filter (FBG2) (Reflectivity $90 \% ; \Delta \lambda=1.4 \mathrm{~nm} ; \lambda=$ $1550.7 \mathrm{~nm}$ ) and the filtered signal was fed into the thermally insulated MZI with path-imbalance of $2 \mathrm{~m}$ using a $50 / 50$ coupler. Three photoreceivers $(40 \mathrm{~V} / \mathrm{mA}$ transimpedance; $125 \mathrm{MHz}$ bandwidth) were used to detect the light from the $3 \times 3$ coupler at the output of the MZI. The output of the photoreceivers were collected using a $250 \mathrm{MHz}$ oscilloscope at a sampling rate of $625 \mathrm{MSa} / \mathrm{s}$. The signal processing procedure is similar to the one described in ${ }^{[6]}$.

Since the sensor's response to dynamic strain has recently been studied ${ }^{[6]}$, this paper focused on characterising the sensor's response to acoustic vibrations. Therefore, a constant $1300 \mathrm{~Hz}$ sinwave with a $4 V_{p p}$ was applied to the disk PZT throughout the experiment. To generate acoustic perturbation, a loud-speaker was used at a distance of $40 \mathrm{~cm}$ from the sensing fibre. To monitor the pressure and the frequency of the sound wave at the fibre, a previously calibrated microphone was installed on the polystyrene sheet in close proximity to the fibre. Sinusoidal voltages with different frequencies were applied to the loud-speaker to generate sound waves with the sound pressure level varying between $85 d B C$ to $115 d B C$. For each experimental run, the backscattered traces were collected for $100 \mathrm{~ms}$.

\section{RESULTS AND DISCUSSION}

The 3D diagram of figure 3 shows the FFT of the phase-detector output for a $450 \mathrm{nc}$ sinusoidal strain at a frequency of $1300 \mathrm{~Hz}$ and a $100 \mathrm{dBC}$ sinusoidal acoustic wave at a frequency of $1500 \mathrm{~Hz}$. Figure 4(a) shows the sensor response to a range of $800 \mathrm{~Hz}$ sinusoidal acoustic wave with different acoustic pressure levels. The vertical axis of this figure which represents the magnitude of the induced strain is drawn in log scale since the acoustic pressure level is expressed in $\mathrm{dB}$. Figure 4(b) shows the frequency response of the sensor (red-line) and that of the microphone (blue-line) to a human voice comprising of many frequencies played through the loud-speaker.

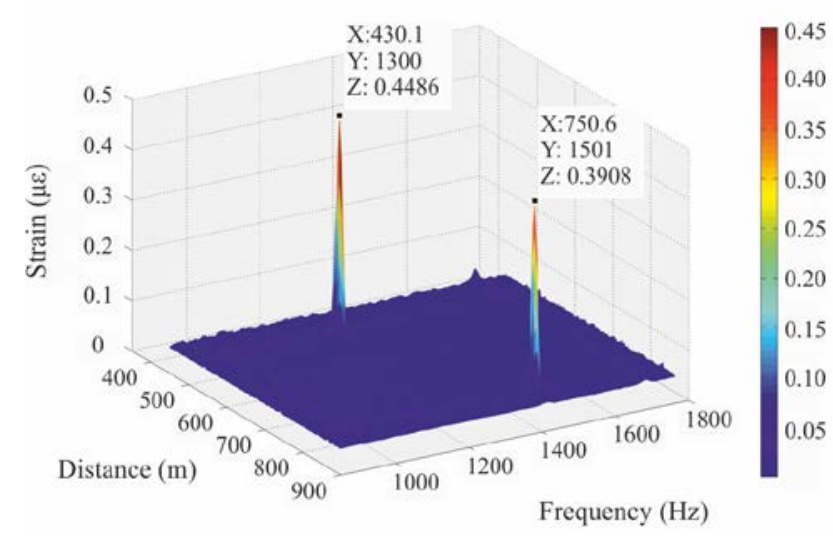

Figure 3: 3D plot of FFT of the phase-detector output for the data points between $400 \mathrm{~m}$ and $900 \mathrm{~m}$.

The two peaks of figure 3 correspond to the two sources of perturbation along the sensing fibre including the disk PZT at $430 \mathrm{~m}$ and the loud-speaker at $750 \mathrm{~m}$. The frequency, location, and the amplitude of the two peaks in this 3D diagram are accurately indicating those of the PZT and the speaker. The sharpness of the peaks indicates the high frequency resolution of the sensor. This resolution is determined by the pulse repetition rate and the total data collection time. For the current experimental setup with a $20 \mu \mathrm{s}$ pulse repetition rate and total data acquisition time of $100 \mathrm{~ms}$, the theoretical frequency resolution of $10 \mathrm{~Hz}$ was calculated. This value was confirmed by the experimental results.

Figure 4(a) shows a linear relationship between the loud-speaker pressure level in $\mathrm{dB}(\mathrm{C})(\mathrm{C}$-weighted pressure level in $\mathrm{dB}$ ) and the $20 \times \log$ of the sensor output for $800 \mathrm{~Hz}$ sinusoidal wave. Similar experiments at other frequencies (i.e.550Hz, $1100 \mathrm{~Hz}$, and $1500 \mathrm{~Hz}$ ) exhibit the same linear characteristic. These results indicate that the amplitude of the sensor output 
is a direct measurement of the applied strain to the sensing fibre and is independent of the applied frequency. The slope of the fitted line is measured to be unity with $\pm 2 \%$ accuracy which confirms the expected linear response within the experimented accuracy. The minimum detectable strain was measured to be $80 \mathrm{n} \varepsilon$ which corresponded to $86 \mathrm{~dB}(\mathrm{C})$ acoustic pressure. The strain accuracy of the sensor was measured to be $50 \mathrm{n} \varepsilon$ which corresponded to an acoustic pressure accuracy of $2.2 \mathrm{~dB}(\mathrm{C})$.

(a)

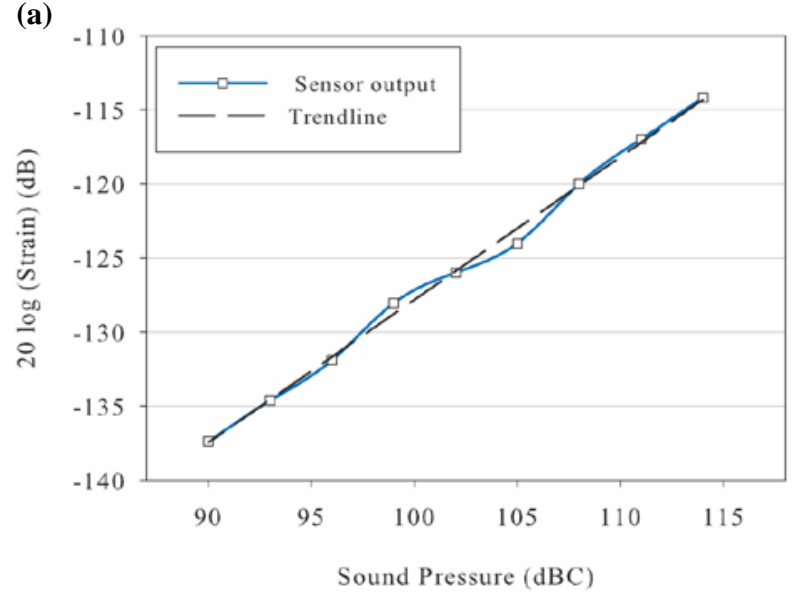

(b)

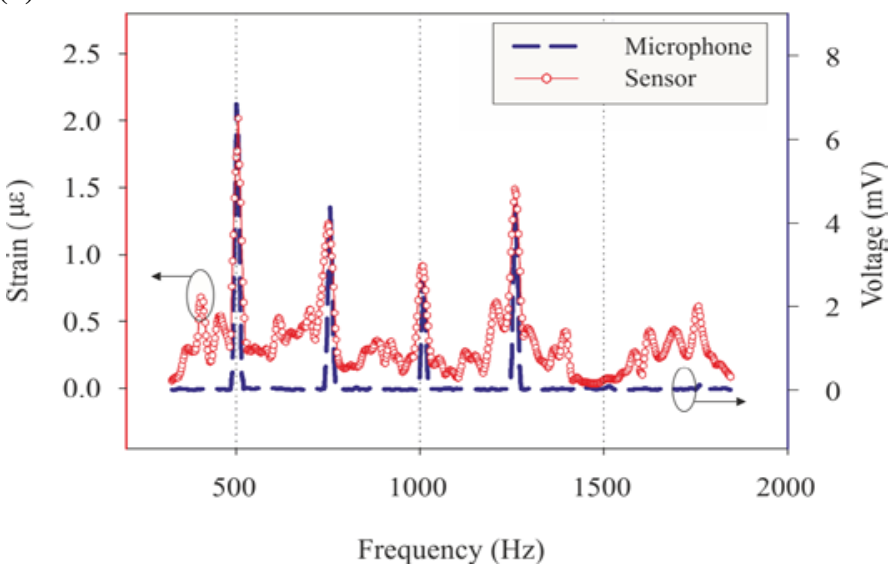

Figure 4: (a) Sensor output versus sound pressure level on the sensing fibre for $800 \mathrm{~Hz}$ sinusoidal acoustic vibration. (b) Frequency response of the sensor (red-line) and that of the microphone (blue-line) to multiple-frequency sound of a human voice played through the loud-speaker for the section of the fibre attached to the polystyrene sheet.

The minimum detectable strain was equal to that previously reported ${ }^{[6]}$ but with improved spatial resolution of $1 \mathrm{~m}$ compared to $2 m$. This improvement was achieved by filtering the phase-detector output using a Chebyshev window.

The frequency response of the sensor output shown in figure 4(b) shows good agreement with that of the microphone. For every frequency component on the microphone output, there is one frequency component on the sensor output which match that of the sensor. However, in addition to a higher noise level, few other frequency components are evident on the FFT of the sensor output. These differences are attributed to the integration of the acoustically induced strain over the spatial resolution of the sensor (i.e. $1 \mathrm{~m}$ ) whilst the microphone output only shows the acoustic vibration in the middle of the polystyrene sheet.

\section{CONCLUSION}

A distributed optical fibre sensor has been demonstrated which uses the $\varphi$-OTDR technique to detect both acoustically induced and dynamic strain perturbations along the sensing fibre. The experimental results show that the demonstrated setup is capable of measuring the frequency and amplitude of multiple dynamic perturbations with a spatial resolution of $1 \mathrm{~m}$ An improved SNR has been demonstrated by introducing a Chebyshev window in the signal processing procedure. In addition, it has been demonstrated that with a frequency resolution of $10 \mathrm{~Hz}$ and a frequency range of $200 \mathrm{~Hz}$ to $5000 \mathrm{~Hz}$, the sensor is capable of detecting and accurately reproducing a multiple-frequency acoustic source such as the acoustic vibration of a human voice.

\section{REFERENCES}

[1] Cole, J. H. et al., “ Fiber optic detection of sound,” J. Acoust. Soc. America, 62, 1136-1138 (1977).

[2] Bucaro, J. A. et al.,, "Fiber optic hydrophone,” J. Acoust. Soc. America, 62, 1302-1304 (1977).

[3] Kurmer, J. P. et al. "Distributed fiber optic acoustic sensor for leak detection," Proc. SPIE 1586, Distributed and Multiplexed Fiber Optic Sensors, 117 (1991).

[4] Taylor, H. F., "Apparatus and method for fiber optic intrusion sensing," U.S. patent 5,194,847, (Jul. 29 1991).

[5] Posey, R. J. et al., "Strain sensing based on coherent Rayleigh scattering in an optical fibre," Electron. Lett., 36, 1688-1689 (2000).

[6] Masoudi, A. et al.,, "Distributed optical fibre dynamic strain sensor based on phase-OTDR," Meas. Sci. Technol., 24(8), (2013).

[7] Priest, R. G., "Analysis of fiber interferometer utilizing 3x3 fiber coupler," IEEE J. Quantum Electron., 18, 1601-1603 (1982). 
[8] Cameron, C. B. et al., "A symmetrical analogue demodulator for optical fiber interferometric sensors," Proc. 34th Midwest symposium on circuits and systems,. 2, 666-671 (1991). 